\title{
Correlación entre el diagnóstico y los hallazgos microscópicos de daño múltiple de órganos, en Morgue Central de Lima, enero a diciembre
}

Kelly Casana, Mary Lapiz, Rosa Carrera, Shérmany Aronés, Mario Castro

Morgue Central de Lima, Instituto de Medicina Legal, Ministerio Público, Lima, Perú

Objetivos: Determinar la correlación entre el diagnóstico macroscópico y los hallazgos microscópicos de daño multiórgano (DMO), en la Morgue Central de Lima, entre enero y diciembre 2010.

Diseño: Descriptivo, observacional, retrospectivo.

Institución: Morgue Central de Lima, Instituto de Medicina Legal, Ministerio Público, Lima, Perú.

Material de estudio: Protocolos de necropsias médico legales.

Intervenciones: Se elaboró matriz con datos de causa de muerte por daño múltiple de órganos, enero a diciembre 2010, con estudio anatomopatológico microscópico.

Principales medidas de resultados: Medidas de tendencia central, frecuencias y porcentajes.

Resultados: Total de muertes por DMO (224); necropsias con $30+$ muestras de órganos 222 (99,1\%), menos de 3 órganos 2 ( $0,9 \%$ ); criterios diagnósticos ( 30 tórganos) de ocho muestras de órganos (bazo, cerebro, corazón, hígado, pulmón, riñón, suprarrenal, tracto gastrointestinal): diagnóstico negativo en muestras $3(1,3 \%)$, diagnóstico 1 muestra 18 (8\%), diagnóstico 2 muestras $40(17,9 \%)$, diagnóstico 30 + muestras $163(72,8 \%)$.

Conclusiones: El diagnóstico se realizó con la positividad del daño en 3 o más órganos afectados; el diagnóstico con daño en 30 + muestras se evidenció en 163 necropsias, que representó un porcentaje de $72,8 \%$, datos reportados en la literatura forense.

Palabras clave: Daño multiórgano.

\section{Asociación de muerte suicida por ahorcadura y pancreatitis hemorrágica, como indicador de toxicidad}

Rosa Carrera, Shérmany Aronés, Denisse Astuhuaman, Kelly Casana, Karina Pariona Instituto de Medicina Legal, Ministerio Público, e Instituto de Patología, UNMSM

Introducción: La asfixia mecánica por ahorcadura es el mecanismo suicida reportado más frecuente, por constricción del cuello ejercido por lazo sujeto a punto fijo, sobre el cual ejerce tracción el peso del cuerpo.

Objetivos: Determinar la incidencia de asociación de muertes violentas por ahorcaduras y, coexistencia de pancreatitis hemorrágica, indicativo de efecto tóxico coadyuvante.

Diseño: Cuantitativo, descriptivo, transversal.

Institución: Instituto de Medicina Legal, Ministerio Público e Instituto de Patología UNMSM.

Material patológico: Informes periciales de necropsias ingresadas a Morgue Central de Lima.

Intervenciones: Medicolegales por denuncia al Ministerio Público por muerte violenta.

Principales medidas de resultados: Medidas de tendencia central porcentual.

Resultados: De 20 necropsias asignadas aleatoriamente para examen anatomopatológico histopatológico complementario, y estudio de patología coexistente, de un total de 233 muertes por ahorcadura, de 567 muertes suicidas, del total de 8833 necropsias realizadas los años 2009-2010. Era masculino 70\% (14), femenino 30\% (06); en 18 con muestra de páncreas, se encontró pancreatitis hemorrágica en 66,7\% (12 casos); tiroiditis crónica 04; linfoma 01, orofaringitis, microesteatosis 2, arterioesclerosis todos, alcoholemia 10\% (23); patología coexistente, cuerpo amarillo gestacional en involución (03).

Conclusiones: Se encontró asociación de ahorcadura y pancreatitis hemorrágica en $66 \%$ de casos, indicador de toxicidad asociada previa al acto, mientras que alcoholemia solo en $10 \%$.

Palabras clave: Ahorcadura, pancreatitis hemorrágica, suicidio. 